\title{
Synchronous asynchronous encounter analysis of runoff between reservoirs based on Copula function
}

\author{
Weidong Zhao, Jing Zhang* \\ College of Water Conservancy, Shenyang Agricultural University, Liaoning Shenyang 110161, China
}

\begin{abstract}
Based on goodness-of-fit test on Copula function, we got the best fitted joint distribution function of runoff between $\mathrm{D}$ and $\mathrm{H}$ reservoirs. Then we analyzed the synchronous asynchronous encounter probability of runoff. The synchronous encounter probability of runoff was $72.46 \%$, while the asynchronous of dryness-wetness or wetness-dryness encounter probability was only $1.06 \%$ between $\mathrm{D}$ and $\mathrm{H}$ reservoirs. The results showed that the runoff conditions were not conducive to the runoff compensation. In order to satisfy the target of water supply, we should also study the reservoir optimization operation, store water in advance and exploit the reservoir regulating potentialities on flood water resources.
\end{abstract}

\section{Introduction}

With the development of economic and the expansion of industrial scale, the contradiction between supply and demand of water resources is becoming more and more prominent. As one of the effective measures, water conveyance project can resolve the uneven problem of water in spatial and temporal distribution and realize the reasonable utilization of water resources, which is becoming the research focus. So it is very important and significant to analyze the synchronous asynchronous encounter probability of runoff between reservoirs in different area and the runoff compensation probability between different basins ${ }^{[1]}$. It can provide important scientific reference for water conveyance projects on water resources reasonable utilization ${ }^{[2]}$.

The central region of Liaoning has a developed economy, dense industrial industry and large population, which plays an important role in promoting the development of Liaoning economy. However, due to the shortage of water resources and the uneven distribution of water resources in spatial and temporal, the quantity of water supply does not match the demand of economic development, which seriously limits the socio-economic development. According to the distribution situation of water resources in Liaoning, the problem of water resources shortage can be solved effectively through the allocation pattern of "East water supplements West" ${ }^{[3]}$.

\footnotetext{
*Corresponding author: zhangjingbluesky@163.com
} 
In this paper, we take $\mathrm{D}$ and $\mathrm{H}$ reservoirs as research objects to discuss the synchronous asynchronous encounter probability of runoff and provide scientific reference for the optimal allocation of water resources and the sustainable utilization of water resources.

\section{Research area and data information}

D reservoir is located in A basin while $\mathrm{H}$ Reservoir is located in $\mathrm{B}$ basin. $\mathrm{H}$ reservoir is the head power station in B basin. Discharge after power generation in $\mathrm{H}$ reservoir will be channeled into D reservoir to satisfy the water demand of central region of Liaoning and alleviate the water shortage problem. In order to analyze the synchronous asynchronous encounter probability of runoff between $\mathrm{D}$ and $\mathrm{H}$ reservoirs, the monthly runoff data from 1980 to 2010 of these two reservoirs had been used.

\section{Research methods}

The problem of synchronous asynchronous encounter probability of runoff is a multidimensional joint probability and conditional probability problem ${ }^{[4]}$. Because the Copula function has a simple model and strong adaptability when solving the multi-variable combined probability distribution, and is superior to the traditional statistical method in the conditional probability calculation ${ }^{[5]}$, we choose Copula function to establish the model of synchronous asynchronous encounter of runoff. Through goodness-of-fit test, the best fitted Copula function model will be selected. Then using the best fitted Copula function model to calculate synchronous asynchronous encounter probability of runoff, we can know the runoff compensation characteristics between $\mathrm{D}$ and $\mathrm{H}$ reservoirs.

\section{Calculation and results}

\subsection{Marginal distribution determination}

We often assume that the hydrological series obey the P-III distribution when we analyze the single variable hydrological data series ${ }^{[6]}$. In this paper, we adopted P-III distribution to express the random variables and marginal distribution when constructing runoff joint distribution model. The probability density function of P-III distribution is as follow:

$$
f(x)=\frac{\beta}{\Gamma(\alpha)}^{\alpha}\left(x-a_{0}\right)^{\alpha-1} e^{-\beta\left(x-a_{0}\right)}
$$

Where $\Gamma(\alpha)$ is Gamma function, $\alpha$ is the shape parameter of P-III distribution, $a_{0}$ is position parameter, $\beta$ is scale parameter, $\alpha, \beta$ are larger than 0 .

$$
\alpha=\frac{4}{C_{s}^{2}}, \quad a_{0}=\bar{x}\left(1-\frac{2 C_{v}}{C_{s}}\right), \quad \beta=\frac{2}{\bar{x} C_{v} C_{S}} .
$$

Where $\bar{x}$ is average value of annual runoff, $C_{s}$ is bias coefficient, $C_{v}$ is discretionary coefficient.

With optimized curve-fitting method, the statistical parameters of annual runoff of D and $\mathrm{H}$ reservoirs were calculated. The results were listed in Table 1. 
Table 1. Statistical parameters of annual runoff of $\mathrm{D}$ and $\mathrm{H}$ reservoirs

\begin{tabular}{ccc}
\hline Statistical parameters & H reservoir & D reservoir \\
\hline $\bar{X}\left(10^{8} \mathrm{~m}^{3}\right)$ & 40.1 & 15.05 \\
$C_{v}$ & 0.54 & 0.82 \\
$C_{S}$ & 2.33 & 2.45 \\
\hline
\end{tabular}

\subsection{Joint distribution determination}

When calculating synchronous asynchronous encounter probability of runoff using Copula function, we should first to estimate the unknown parameter in Copula function. According to the past studies, the maximum likelihood estimation method, non-parameter method, and semi-parameter method were mostly used. Among them, the non-parameter method had been chosen by many scholars because of the advantages of its simple process and accurate results. So in this paper, we used non-parameter method to estimate the unknown parameter, which was $\theta$, in Copula function. The principle of non-parameter method was estimating the parameter by the mathematical relationship between Kendall $\tau$ and the parameter which would be estimated in Copula functions ${ }^{[7]}$. Based on the observed runoff data $\left(x_{n}, y_{n}\right)$, the equation to calculate Kendall $\tau$ was as follow:

$$
\begin{gathered}
\hat{\tau}=\frac{2}{n(\mathrm{n}-1)} \sum_{i=1}^{n} \sum_{j=1}^{n} \operatorname{sign}\left[\left(x_{i}-x_{j}\right)\left(y_{i}-y_{j}\right)\right] \\
\operatorname{sign}=\left\{\begin{array}{l}
1, x_{i}>x \\
0, x_{i}=x_{j} \\
-1, x_{i}<x_{j}
\end{array}\right.
\end{gathered}
$$

Where $n$ represented the sample size; $\hat{\tau}$ was the estimated value of Kendall rank correlation coefficient.

Based on the runoff data of 31 years of $\mathrm{D}$ and $\mathrm{H}$ reservoirs, we calculated the Kendall rank correlation coefficient which was 0.673 . In order to get the best fitted Copula function model, we selected 6 Copula function models. The 6 Copula function models and their respectively estimated parameter value were shown in table 2.

\begin{tabular}{|c|c|c|c|c|}
\hline No. & Copula function model & Generator & $\begin{array}{c}\text { Relationship } \\
\text { between } \tau \text { and } \\
\theta\end{array}$ & $\begin{array}{c}\text { Estimated } \\
\text { value of } \\
\theta\end{array}$ \\
\hline 1 & $\max \left[\left(u^{-\theta}+v^{-\theta}-1\right), 0\right]$ & $\frac{1}{\theta}\left(t^{-\theta}-1\right)$ & $\frac{\theta}{\theta+2}$ & 4.12 \\
\hline 2 & $\max \left(1-\left[(1-u)^{\theta}+(1-v)^{\theta}\right]^{\frac{1}{\theta}}, 0\right)$ & $(1-t)^{\theta}$ & $\frac{\theta-2}{\theta}$ & 6.12 \\
\hline 3 & $E X P^{-\left[(-\ln u)^{\theta}+(-\ln v)^{\theta}\right]^{\frac{1}{\theta}}}$ & $(-\ln t)^{\theta}$ & $\frac{\theta-1}{\theta}$ & 3.06 \\
\hline 4 & $1+\left[\left(u^{-1}-1\right)^{\theta}+\left(v^{-1}-1\right)^{\theta}\right]^{\frac{1}{\theta}}$ & $\left(\frac{1}{t}-1\right)^{\theta}$ & $1-\frac{2}{3 \theta}$ & 2.04 \\
\hline
\end{tabular}

Table 2. Copula function models and their estimated parameter values 


$$
\begin{aligned}
& 5\left\{1+\left[\left(u^{-\frac{1}{\theta}}-1\right)^{\theta}+\left(v^{-\frac{1}{\theta}}-1\right)^{\theta}\right]^{\frac{1}{\theta}}\right\}^{-\theta}\left(t^{-\frac{1}{\theta}}-1\right)^{\theta} \quad 1-\frac{4}{2+4 \theta} \quad 2.56 \\
& 6 \max \left(\left\{1-\left[\left(1-u^{\frac{1}{\theta}}\right)^{\theta}+\left(1-v^{\frac{1}{\theta}}\right)^{\theta}\right]^{\frac{1}{\theta}}\right\}^{\theta}, 0\right) \quad\left(1-t^{\frac{1}{\theta}}\right)^{\theta} \quad 1+\frac{2}{1+2 \theta} \quad 3.56
\end{aligned}
$$

Through goodness-of-fit test, we selected the best fitted Copula function model from the 6 Copula function models. In this paper, we used three indicator values to select the best fitted Copula function model, which were $R M S E, A I C$ and $B I C^{[8]}$. If one of the 6 Copula function models whose three indicator values were all the smallest, the corresponding Copula function model was the best fitted model.

The equation to calculate $R M S E$ was as follow:

$$
R M S E=\sqrt{\frac{1}{n} \sum_{i=1}^{n}\left[P_{c}(i)-P_{o}(i)\right]^{2}}
$$

Where $n$ was sample size, $P_{c}$ was the theoretical frequency value of two-dimensional joint distribution, $P_{0}$ was the experienced frequency value of two-dimensional joint distribution. The equation to calculate experienced frequency of two-dimensional joint distribution was as follow ${ }^{[9] .}$

$$
P_{o}=P\left(x \leq x_{i}, \quad \mathrm{y} \leq y_{i}\right)=\frac{\sum_{m=1}^{n} \sum_{n=1}^{n} N_{m n}-0.44}{N+0.12}
$$

Where $N_{m n}$ represented the data number when $x \leq x_{i}$ and $y \leq y_{i} ; N$ was the total number of data.

The equations to calculate $A I C$ and $B I C$ were as follows ${ }^{[8]}$.

$$
\begin{aligned}
A I C & =n * \ln (M S E)+2 * m \\
B I C & =n * \ln (M S E)+m * \ln n \\
M S E & =\frac{1}{n-m} \sum_{i=1}^{n}\left[p_{c}(i)-p_{o}(i)\right]^{2}
\end{aligned}
$$

Where $m$ was the estimated value of unknown parameter in Copula function model.

The results on three indicator values among 6 Copula function models were listed in table 3. According to table 3, we knew that the three indicator values of the sixth Copula function were the smallest, which indicated that the sixth Copula function model was the best fitted function.

Table 3. Indicator values for 6 Copula function models

\begin{tabular}{cccc}
\hline No. & RMSE & AIC & BIC \\
\hline 1 & 0.082 & -152.233 & -150.799 \\
2 & 0.076 & -156.790 & -155.356 \\
3 & 0.076 & -156.382 & -154.948 \\
4 & 0.079 & -154.388 & -152.954 \\
5 & 0.078 & -155.534 & -154.100 \\
6 & 0.076 & -156.920 & -155.486 \\
\hline
\end{tabular}


The comparison on runoff joint distribution frequency between experienced values and theoretical values which were calculated based on the best fitted Copula function was shown in figure1. From figure 1, we also knew that the sixth Copula function had good fitness and could be used to calculate synchronous asynchronous encounter probability of runoff between $\mathrm{D}$ and $\mathrm{H}$ reservoirs.

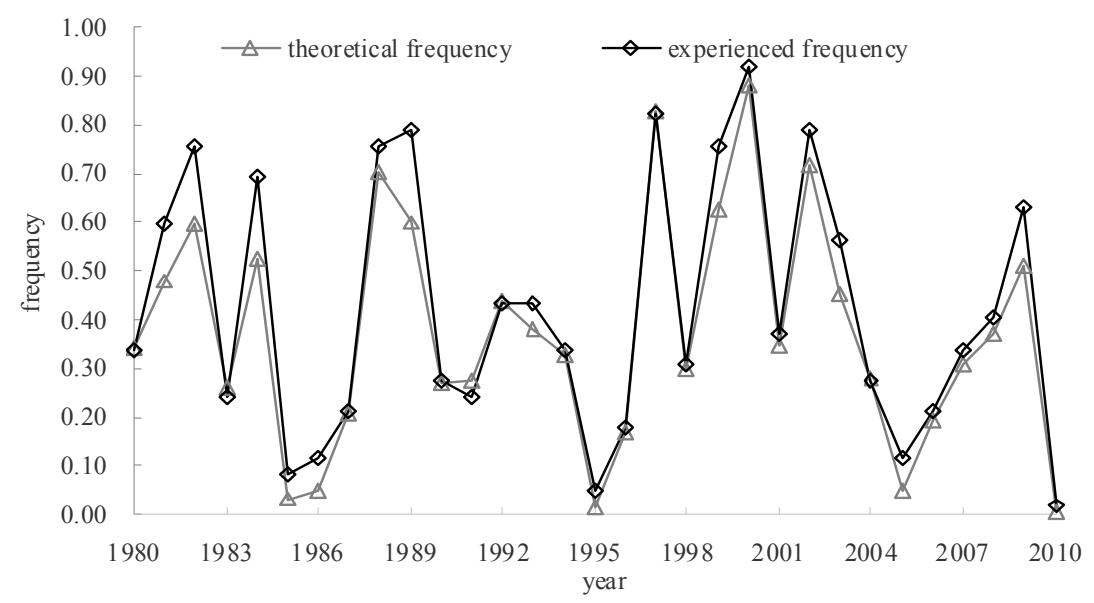

Fig.1 comparison on runoff joint distribution frequency between experienced and theoretical values

\subsection{Synchronous asynchronous encounter probability of runoff calculation}

According to the reference [10], we took the frequency of $37.5 \%$ and $62.5 \%$ as the standard to divide the wetness year, median water year and dryness year. Through the hydrologic frequency curve of $\mathrm{D}$ and $\mathrm{H}$ reservoirs, we got the runoff quantity for different frequency, which were listed in table 4.

Table 4. Runoff quantity for different typical frequency for D and $\mathrm{H}$ reservoirs Unit: $10^{8} \mathrm{~m}^{3}$

\begin{tabular}{ccc}
\hline reservoir & Wetness year $(37.5 \%)$ & Dryness year $(62.5 \%)$ \\
\hline $\mathrm{D}$ & 13.89 & 8.37 \\
$\mathrm{H}$ & 38.48 & 28.41 \\
\hline
\end{tabular}

According to the best fitted Copula function, we calculated synchronous asynchronous encounter probability of runoff between $\mathrm{D}$ and $\mathrm{H}$ reservoirs. There were 9 synchronous asynchronous encounter conditions of runoff between $\mathrm{D}$ and $\mathrm{H}$ reservoirs, which were listed as follows:

Wetness year in D reservoir and wetness year in $\mathrm{H}$ reservoir:

$P(x \geq 13.89, y \geq 38.48)$;

Median water year in D reservoir and median water year in $\mathrm{H}$ reservoir:

$P(8.37<x<13.89,28.41<y<38.48)$;

Dryness year in D reservoir and dryness year in $\mathrm{H}$ reservoir:

$P(x \leq 8.37, y \leq 28.41)$;

Wetness year in D reservoir and median water year in $\mathrm{H}$ reservoir:

$P(x \geq 13.89,28.41<y<38.48)$;

Wetness year in $\mathrm{H}$ reservoir and median water year in D reservoir:

$P(y \geq 38.48,8.37<x<13.89)$; 
Wetness year in D reservoir and dryness year in $\mathrm{H}$ reservoir:

$P(x \geq 13.89, y \leq 28.41)$;

Wetness year in $\mathrm{H}$ reservoir and dryness year in $\mathrm{D}$ reservoir:

$P(y \geq 38.48, x \leq 8.37)$;

Median water year in $\mathrm{D}$ reservoir and dryness year in $\mathrm{H}$ reservoir:

$P(8.37<x<13.89, y \leq 28.41)$;

Median water year in $\mathrm{H}$ reservoir and dryness year in $\mathrm{D}$ reservoir:

$P(28.41<y<38.48, x \leq 8.37)$

The synchronous asynchronous encounter probability to each encounter type was calculated, the results were shown in table 5.

Table 5. Synchronous asynchronous encounter probability between $\mathrm{D}$ and $\mathrm{H}$ reservoirs

\begin{tabular}{ccccc}
\hline \multicolumn{2}{c}{ Encounter probability of } & \multicolumn{3}{c}{ H reservoir } \\
\cline { 2 - 5 } & runoff & Wetness & Median & Dryness \\
\hline \multirow{2}{*}{$\mathrm{D}$} & Wetness & 31.01 & 5.43 & 1.06 \\
\multirow{2}{*}{ reservoir } & Median & 5.43 & 12.30 & 7.27 \\
& Dryness & 1.06 & 7.27 & 29.16 \\
\hline
\end{tabular}

From table 5, we knew that the synchronous encounter probability between $\mathrm{D}$ and $\mathrm{H}$ reservoirs was $72.46 \%$ and the dryness-dryness encounter probability was $29.16 \%$, while the asynchronous encounter probability was $27.54 \%$ and the dryness-wetness or wetnessdryness encounter probability was $1.06 \%$ between $\mathrm{D}$ and $\mathrm{H}$ reservoirs. The results showed that the runoff conditions between $\mathrm{D}$ and $\mathrm{H}$ reservoirs were not conducive to the runoff compensation.

\section{Conclusions}

According to the theory of Copula function, we selected the best fitted Copula function model for $\mathrm{D}$ and $\mathrm{H}$ reservoirs. Based on the best fitted Copula function model, we analyzed the synchronous asynchronous encounter probability between $\mathrm{D}$ and $\mathrm{H}$ reservoirs. The calculation results showed that the probability of synchronous encounter between $\mathrm{D}$ and $\mathrm{H}$ reservoirs was $72.46 \%$, while dryness-wetness or wetness-dryness encounter probability was only $1.06 \%$, which was not conducive to the runoff compensation between reservoirs. In order to satisfy the water demand of Liaoning, each reservoir needs to study reservoir optimization operation and increase the utilization of flood water resources, and make full use of reservoir regulation and storage capacity on flood water resources.

\section{References}

[1] H.H. Mao, J.Z. Li, X.Y. Wang. Analysis on wet-dry and compensative characteristics of regional rainfall. Journal of Tianjin University. Vol.42(5),377-381, (2009)

[2] Y. Wang, Q. Wang. Study on runoff compensation characteristics in rainy and dry season for the water conveyance project based on Copula function. Water resources \& hydropower of Northeast. 07,37-39. (2018)

[3] Z.H. Zhang, Q. Zhang, M. Z. Xiao, et al. Reservoir-based ecological water operation in the Liaohe River Basin characterized by synchronous occurrence of wet/dry events. Acta Ecologica Sinica, 36(7), 2024-2033. (2016) 
[4] H. Xie, Q. Luo, J.S. Huang. Synchronous asynchronous encounter analysis of multiple hydrologic regions based on 3D copula function. Advances in water science. Vol 23(2), 186-193. (2012)

[5] Q.L. Xiong, X.C. He, L. Kang. Synchronous asynchronous encounter probability of rich poor precipitation between water source area and water receiving area based on copula theory in middle route of south to north water transfer project. Water resources and power. Vol.27(6), 9-11,112. (2009)

[6] J.Y. Niu, P. Feng, Z.H. Ding. Study of the wetness-dryness compensation characteristics of Luan River diversion reservoir's inflow based on multivariate copula functions. Journal of Jilin University (Earth Science Edition). Vol39,(6), 1095-1100. (2009) [7] C. Gennst, L P. Rwest. Statistical inference procedures for bivariate archimedean copula. Journal of the Amercian Statistical Association, 88(423): 1034-1043. (1993)

[8] S.P. Song, H.J. Cai, J. L. Jin, et al. Copulas function and its application in hydrology. Beijing: Science Press. (2012)

[9] L. Zhang, P. Vijay Singh Bivariate rainfall frequency distributions using Archimedean copulas.Journal of Hydrology. 332(1-2). 93-109. (2007)

[10] H.X. Zheng, C.M. Liu. Analysis on asynchronism-synchronism of regional precipitation in planned South-to-North water transfer areas. Acta Geographica Sinica. (5), 523-532. (2000) 